\title{
Graphene Supercapacitor as a Voltage Controlled Saturable Absorber for Femtosecond Pulse Generation
}

\author{
Isinsu Baylam ${ }^{1,2}$, M. Natali Cizmeciyan ${ }^{1,2}$, Sarper Ozharar $^{3}$, E. Ozan Polat ${ }^{4}$, \\ Coskun Kocabas ${ }^{4}$, and Alphan Sennaroglu ${ }^{1,2, *}$ \\ ${ }^{1}$ Laser Research Laboratory, Departments of Physics and Electrical-Electronics Engineering, Koç University, \\ Rumelifeneri, Sarlyer, Istanbul 34450, Turkey. \\ ${ }^{2}$ Koç University Surface Science and Technology Center (KUYTAM), Rumelifeneri, Sarlyer, Istanbul 34450, Turkey. \\ ${ }^{3}$ College of Arts and Sciences, Bahçeşehir University, Beşiktaş, Istanbul 34353, Turkey. \\ ${ }^{4}$ Bilkent University, Department of Physics, Ankara 06800, Turkey. \\ *asennar@ku.edu.tr
}

\begin{abstract}
For the first time to our knowledge, we employed a graphene supercapacitor as a voltage controlled saturable absorber at bias voltages of $0.5-1 \mathrm{~V}$ to generate $84-\mathrm{fs}$ pulses from a solid-state laser near $1255 \mathrm{~nm}$.

OCIS codes: (140.4050) Mode-locked lasers; (140.7090) Ultrafast lasers
\end{abstract}

A monolayer graphene which has a nearly constant absorption over a broad wavelength range has been shown to work as an effective saturable absorber for lasers operating in the near and mid infrared [1-3]. One drawback is that even for monolayer graphene, the small-signal round-trip insertion loss is of the order of $5 \%$, which makes it quite challenging to operate low-gain lasers with graphene saturable absorbers. One approach is to use graphene-based capacitor architectures in which the Fermi level can be shifted to reduce the passive losses, while maintaining a sufficient level of nonlinearity to initiate mode-locked operation. Graphene-based solid-state capacitors of this kind have been shown to operate with variable level of absorption, but their operation was limited to the infrared wavelengths $[4,5]$. Among the possible capacitor architectures, it has been shown that graphene-based supercapacitors posses the superior property that, due to the use of a high-dielectric-constant electrolyte, the Fermi level can be readily shifted to vary the absorption up to the visible region even with low bias voltages of the order of a few volts [6].

Here, we report the first demonstration of a graphene supercapacitor operated as a voltage controlled graphene saturable absorber (VCG-SA) at bias voltages below 3V. Inside the resonator of a Cr:forsterite laser operated at $1255 \mathrm{~nm}$, the voltage of the VCG-SA was varied in the 0-3V range to determine the optimum operating conditions. Mode-locked operation could be produced for bias voltages in the range of $0.5-1 \mathrm{~V}$. At the bias voltage of $1 \mathrm{~V}, 84$-fs pulses with a time-bandwidth product of 0.32 and peak power of $37 \mathrm{~kW}$ were generated at the wavelength of 1255 $\mathrm{nm}$. We also investigated the dependence of the saturation fluence, modulation depth, and linear loss of the VCGSA on the applied voltage.
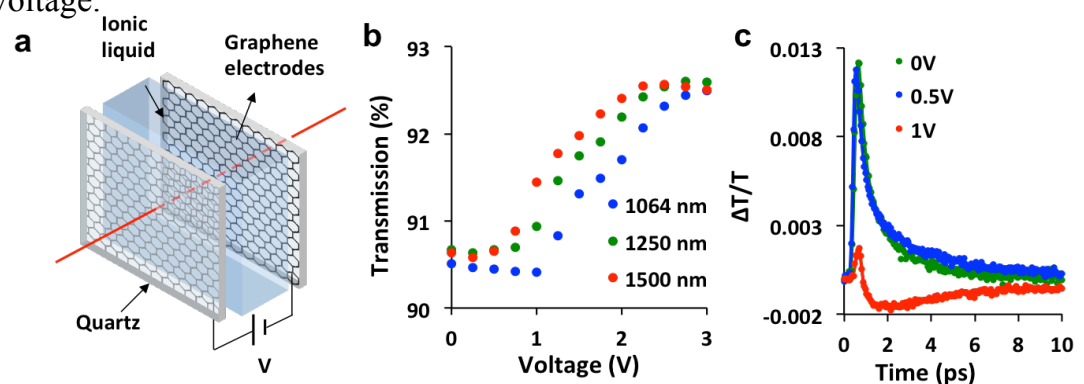

Fig.1. (a) Schematic of the graphene-based supercapacitor. (b) The measured transmission of the device at normal incidence as a function of the bias voltage for different wavelengths (c) Ultrafast response of the graphene-based supercapacitor at the probe wavelength of $1250 \mathrm{~nm}$ for different bias voltages.

To fabricate the graphene supercapacitor, two monolayer large area graphene samples were first synthesized by using chemical vapor deposition and transfer-printed to quartz substrates. The spacing between the two graphene electrodes was then filled with a high-dielectric-constant electrolyte (Diethymethyl (2-methoxyethyl) ammonium bis (trifluromethylsulfony) imide) as shown in Fig. 1(a). With this electrolyte, the Fermi level can be shifted by up to $2.3 \mathrm{eV}$ at a voltage bias of only $3 \mathrm{~V}$ enabling the use of this supercapacitor as a modulator up to $450 \mathrm{~nm}$ in the visible range. The VCG-SA operates as a fast saturable absorber and has a broadband absorption under zero bias. When a voltage is applied, the Fermi level of the graphene layers begins to shift and eventually, the absorption of the device is completely suppressed for wavelengths with photon energy below $2 \mid \mathrm{E}_{\mathrm{F}} \mathrm{I}$ due to Pauli blocking [6]. This effect can be seen in Fig. 1(b), which shows the measured transmission of the device at normal incidence for the wavelengths 
of 1064, 1250, and $1500 \mathrm{~nm}$. Pump-probe measurements depicted in Fig. 1(c) further show that, up to an applied voltage of $1 \mathrm{~V}$, the typical ultrafast response of the graphene can be seen with average fast and slow time constants of $283 \mathrm{fs}$ and $1.9 \mathrm{ps}$. For voltages beyond $1 \mathrm{~V}$, however, the device has more complicated dynamics, possibly because intra-band transitions start to dominate over inter-band transitions at $1250 \mathrm{~nm} \mathrm{[7].} \mathrm{Our} \mathrm{measurements} \mathrm{further} \mathrm{showed}$ that the saturation fluence at $1250 \mathrm{~nm}$ monotonically increased with applied bias, becoming $456 \mu \mathrm{J} / \mathrm{cm}^{2}$ at $1 \mathrm{~V}$. Furthermore, the modulation depth was found to decrease from $1.09 \%$ at $0 \mathrm{~V}$ to $0.69 \%$ at $1 \mathrm{~V}$ at $1250 \mathrm{~nm}$. In the mode-locking experiments, it was not possible to use this device as an effective saturable absorber near $1255 \mathrm{~nm}$ for bias voltages above $1 \mathrm{~V}$.
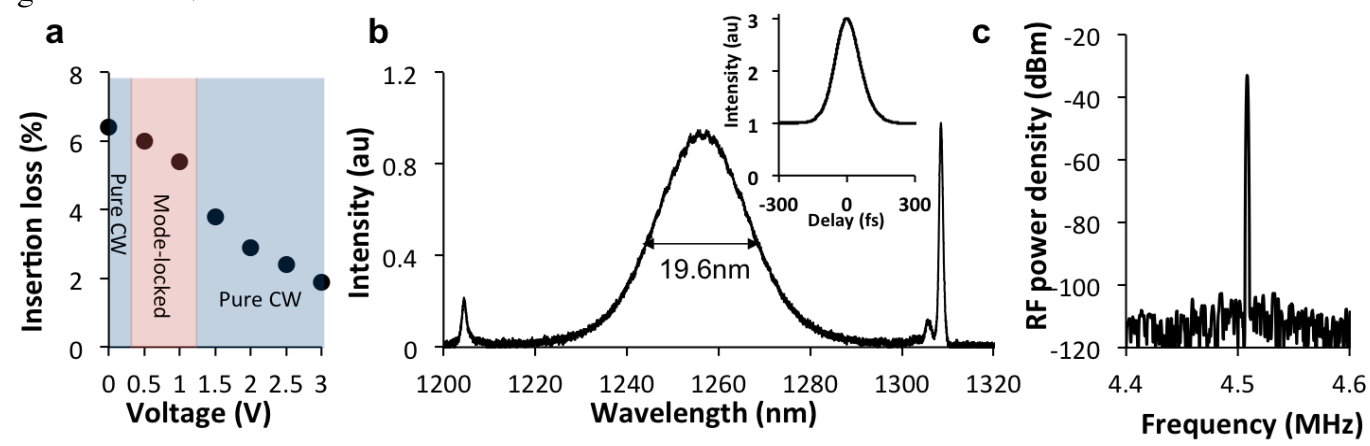

Fig.2. (a) Measured single-pass insertion loss of the graphene based supercapacitor device at $1255 \mathrm{~nm}$ as a function of the bias voltage (b) The spectrum and autocorrelation trace (inset) of the generated pulses at $1255 \mathrm{~nm}$ at the bias voltage of $1 \mathrm{~V}$. (c) The corresponding RF spectrum of the pulses at $1 \mathrm{kHz}$ resolution bandwidth. The pulse repetition frequency was $4.51 \mathrm{MHz}$.

For mode-locking experiments, we used a multipass-cavity $1250 \mathrm{~nm} \mathrm{Cr}^{4+}$ :forsterite laser operated at room temperature similar to the setup used in [8]. Figure 2 summaries the power performance and the mode-locking results obtained by using the VCG-SA. The variation of the single-pass insertion loss of the device, determined from threshold pump power measurements, is shown in Fig. 2(a). As can be seen here, as the applied voltage is increased, the shift in the Fermi level reduces the passive losses. For voltages below $0.5 \mathrm{~V}$, the loss level of the device was relatively high and the intracavity energy of the resonator was not sufficient to support mode-locked operation at the pump power of $7 \mathrm{~W}$. Although the laser could be operated with high continuous-wave output powers for applied voltages above $1 \mathrm{~V}$, mode-locked operation could not be obtained again, due to the fact that the fast saturable absorber action of the device gets suppressed above $1 \mathrm{~V}$ as discussed earlier with reference to Fig. 1(c). For bias voltages between $0.5 \mathrm{~V}$ and $1 \mathrm{~V}$, mode-locked operation of the laser could be successfully obtained. At the bias voltage of $1 \mathrm{~V}$, the laser produced 84 -fs pulses with a time-bandwidth product of 0.32 and $37 \mathrm{~kW}$ of peak power. Figures 2(b) and 2(c) show the mode-locked spectrum, autocorrelation trace (inset), and the RF spectrum of the generated pulses at the bias voltage o $1 \mathrm{~V}$.

In conclusion, we report, for the first time to our knowledge, the use of a graphene-based supercapacitor saturable absorber for femtosecond pulse generation from a solid-state laser. A unique advantage of the graphene supercapacitor, in comparison with its solid-state counter parts, is the possibility of shifting the Fermi level by several $\mathrm{eV}$ (in our case $2 \mathrm{IE}_{\mathrm{F}} \mathrm{l}$ by as much as $2.3 \mathrm{eV}$ ) with relatively low bias voltages of the order of several volts. Furthermore, the device can be fabricated by using simple and low-cost procedures. In our experiments conducted with a multi-pass cavity $\mathrm{Cr}^{4+}$ :forsterite laser at $1255 \mathrm{~nm}$, we could successfully initiate femtosecond pulse generation and obtained 84 -fs pulses with a time-bandwidth product of 0.32 and peak power of $37 \mathrm{~kW}$ at the bias voltage of $1 \mathrm{~V}$. We believe that the voltage controlled graphene saturable absorber device (VCG-SA) has the potential to be implemented in a wide range of lasers operating in the visible and near infrared spectrum.

\section{References}

[1] I. H. Baek et al., "Efficient Mode-Locking of Sub-70-fs Ti:Sapphire Laser by Graphene Saturable Absorber," Appl. Phys. Express 5, 032701, (2012).

[2] Z. Sun, T. Hasan, and A. C. Ferrari, "Ultrafast lasers mode-locked by nanotubes and graphene," Physica E 44, 1082-1091, (2012).

[3] M. N. Cizmeciyan et al., "Graphene mode-locked femtosecond Cr:ZnSe laser at 2500nm," Opt. Lett. 38, 341-343, (2013).

[4] C. C. Lee et al., "Broadband graphene electro-optic modulators with sub-wavelength thickness," Opt. Express 20, 5264-5269, (2012).

[5] C. C. Lee et al., "Frequency comb stabilization with bandwidth beyond the limit of gain lifetime by an intracavity graphene electrooptic modulator," Opt. Lett. 37, 3084-3086, (2012).

[6] E. O. Polat and C. Kocabas, "Broadband Optical Modulators Based on Graphene Supercapacitors," Nano Lett. 13, 5851-5857, (2013).

[7] L. M. Malard et al., "Observation of intra and interband transitions in the transient optical response of graphene," New J. Phys. 15, (2013).

[8] S. Ozharar et al., "Graphene mode-locked multipass-cavity femtosecond $\mathrm{Cr}^{4+}$ : forsterite laser," J. Opt. Soc. Am. B 30, 1270-1275, (2013). 